\title{
A Brief History of Near-Field Measurements of Antennas at the National Bureau of Standards
}

\author{
RAMON C. BAIRD, SENIOR MEMBER, IEEE, ALLEN C. NEWELL, SENIOR MEMBER, IEEE, AND \\ CARL F. STUBENRAUCH, SENIOR MEMBER, IEEE
}

\section{Invited Paper}

\begin{abstract}
The National Bureau of Standards (NBS) played a pioneering role in the development of practical planar near-field antenna measurement techniques. A brief history is presented of that role, which began with theoretical studies to determine corrections for diffraction in a microwave measurement of the speed of light. NBS contributions to the development of nonplanar near-field measurement theory and practice are also described.
\end{abstract}

\section{EARLY History}

$\mathrm{T}$ HE development of accurate near-field measurements for antennas by the National Bureau of Standards (NBS) is a superb example of how research in one area can lead to unexpected and important results in another area. In about 1955 a project to determine the speed of light with a microwave analog of the Michelson interferometer was initiated at NBS [1], [2]. Fig. 1 is a simplified diagram of the interferometer. A single hybrid junction splits a microwave signal into two paths and recombines the reference signal with the signal reflected from the moveable mirror to produce an interference signal at the detector output. The mirror displacement between successive minima is $\lambda_{e} / 2$, where $\lambda_{e}$ is the effective wavelength and is determined by accurately measuring the mirror displacement required to produce a known number of minima. The product of the wavelength and the frequency then determines the speed of light.

Although simple in concept, the experiment demanded extreme attention to detail and the values obtained required two principal corrections. The first was due to the refractive index of air. The second was a diffraction correction associated with the fact that the mirror was located in the near field of the antenna and was not illuminated by plane waves. The radiated beam actually diverged slightly; this resulted in a measured $\lambda_{e}$ which, in the NBS arrangement, was longer than the true wavelength by about two parts in $10^{5}$. Since the overall accuracy goal for the speed of light was about three parts in $10^{7}$, the key to achieving this goal was an accurate diffraction correction. In 1954 Dayhoff conceived a theoretical approach that would lead to the solution of this problem. He discussed these concepts with Kerns, who immediately became interested. Together they developed a rigorous diffraction theory [3] for making such corrections. This theory uses the plane-wave scattering matrix (PWSM) formalism, and its application requires measurements of relative ampli-

Manuscript received February 29, 1988.

The authors are with the Electromagnetic Fields Division, National Bureau of Standards, Boulder, CO 80303.

IEEE Log Number 8821096 tude and phase of the radiated fields over an aperture plane close to the antenna. Taking the inverse Fourier transforms of these data allows one, after a rather complicated bit of computer processing, to determine an accurate correction for the measured wavelength.

The experiment was progressing well and all accuracy objectives were being met except for the diffraction correction. The computer programs needed refining, and more closely spaced data may have been required. We were confident of eventual success, but at this time the laser was invented. Clearly, the laser would provide much greater accuracy for the speed of light than we could ever hope to achieve with this microwave technique, so the project was abandoned.

Fortunately, Kerns recognized that his diffraction theory had other applications. He said in his introduction to the diffraction paper [3]:

Inasmuch as we wish to provide a theory inherently capable of dealing with high-accuracy experiments, a considerable degree of generality is required to avoid over-idealization. It is interesting to note that the required general reflection system could equally well represent a system consisting of radar and target, for example; similarly, the general transmission system could represent a point-to-point communication system. In view of the generality of the basic arrangments it seems likely that applications of the theory will also be found in problems other than the ones that motivated this work.

This statement indeed proved to be prophetic. In fact, Kerns discussed the possibility of probe-corrected near-field antenna measurements based on this new theory at the 1963 Spring URSI Meeting in Washington, DC [4].

In late 1963 NBS was approached by the Advanced Research Projects Agency (ARPA) of the Defense Department to undertake the development of microwave standards needed for radar systems. ARPA representatives pointed out that the practical development of large antennas and arrays for radar, tracking, and satellite systems had created a need for highly accurate far-field pattern determinations and gain measurements. However, the far-field distances of such antennas were usually too large to allow the use of conventional ranges so ARPA was interested in alternative methods for measuring antenna performance. Kerns realized that his diffraction theory could be applied in a very general sense to antenna measurement problems and proposed that NBS undertake the

U.S. Government work not protected by U.S. copyright 


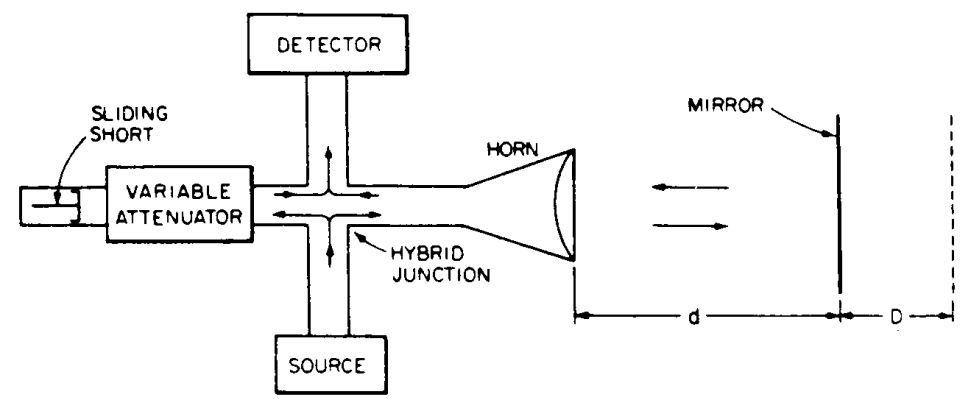

Fig. 1. Simplified diagram of Michelson interferometer used in microwave measurement of speed of light.

task of evaluating the planar near-field scanning (PNFS) method as an accurate and practical measurement tool. ARPA accepted the proposal, and a project to determine the feasibility of predicting antenna far-field performance from measured near-field data was included in the radar standards program.

Several important tasks were addressed by this ARPA project. 1) A small partially automated near-field scanner and digital data recording system were developed. 2) The "diffraction theory" was analyzed and adapted to antenna measurements. 3) Preliminary computer programs were written to calculate far-field patterns from near-field data. 4) Near-field measurements were performed at $48 \mathrm{GHz}$ on the high-gain antenna used for the speed of light experiment. 5) These data were used to obtain preliminary estimates of the optimum data point spacing, to examine the analytical procedures, and to perform checks on the computer programs. Computed farfield patterns demonstrated the feasibility of the approach but also indicated the need to refine the analytical procedures and computer programs. A larger scanner would also be required for the technique to become practical for measuring a variety of common antennas.

About this time NBS management realized there was a growing need for accurate standards and measurement techniques for electromagnetic field and antenna parameters, so the Fields and Antennas Section was organized in April 1966. The objective of this group was to develop means of performing reliable measurements of specifiable and high accuracy. NBS did not have a far-field range and the measurement problems associated with far-field measurements were apparent, so we decided to concentrate on the development of near-field techniques. Moreover, conventional techniques rely upon comparison with a standard antenna, so even if all other errors could be brought under control the problem of accurately evaluating the standard would remain. A different approach must be used to characterize the standard, and this approach should involve a minimum number of assumptions about the antenna and test facility.

These problems are largely overcome with the near-field scanning and extrapolation methods developed by NBS. Both methods are based on the PWSM formulation of Kerns, which we believe is the most general, complete, and useful theoretical formulation of the planar problem. It is put forth as a basis for advanced antenna theory, especially as it relates to antenna measurements. It provides a complete description of general antennas and permits analysis of antenna-antenna interactions at arbitrary distances. Expressions for asymptotic quantities such as gain, effective area, polarization, and far-field patterns are readily obtained in terms of the scattering parameters. The theory is essentially free of restrictive assumptions and built-in approximations, and the method is applicable to any type of directive antenna including phased arrays. (Practical implementation does require that multiple reflections between the antennas be neglected.) It is important to note that this was the first theoretical formulation to include a means of rigorously correcting for the effects of an arbitrary measurement probe.

The early theoretical and experimental work was documented in unpublished reports to ARPA (1964-1967) and presented in various lectures and talks. Between 1964 and 1970 the PNFS theory was essentially completed and published along with the first NBS measurement results and computed far-field patterns [5]-[7].

In 1969 Wacker extended the PWSM theory of Kerns to derive the theoretical foundation for the extrapolation method [8]. This technique, when coupled with the generalized threeantenna measurement method [9], is the most accurate method known for determining absolute gain and polarization of directional antennas. It requires a minimum of a priori information about the antennas and, in its fully developed form, provides the basis for all NBS standard antenna calibrations [10].

\section{Implementation and Dissemination of Planar Technique}

The practical implementation of the planar near-field measurement technique was accomplished at NBS through a series of research efforts on various types of antennas. In many cases, these efforts were sponsored in part by organizations desiring to apply the near-field measurements to special antenna problems. Our first measurements were performed on the millimeter-wave antenna that had been a part of the velocity-of-light experiment. The measurement system shown in Fig. 2 was used from about 1963 to 1974, could measure over an area $1.2 \mathrm{~m}$ on each side, and required significant manual operation. In spite of its limitations, a number of measurements were completed on a variety of antennas operating at frequencies between 50 and $65 \mathrm{GHz}$. These tests were used to develop the basic measurement techniques and determine the requirements of the measurement system. For instance, the theory as developed by Kerns [11] had used the sampling theorem and the band limiting of the plane-wave spectrum due to evanescent modes to predict that incremental 


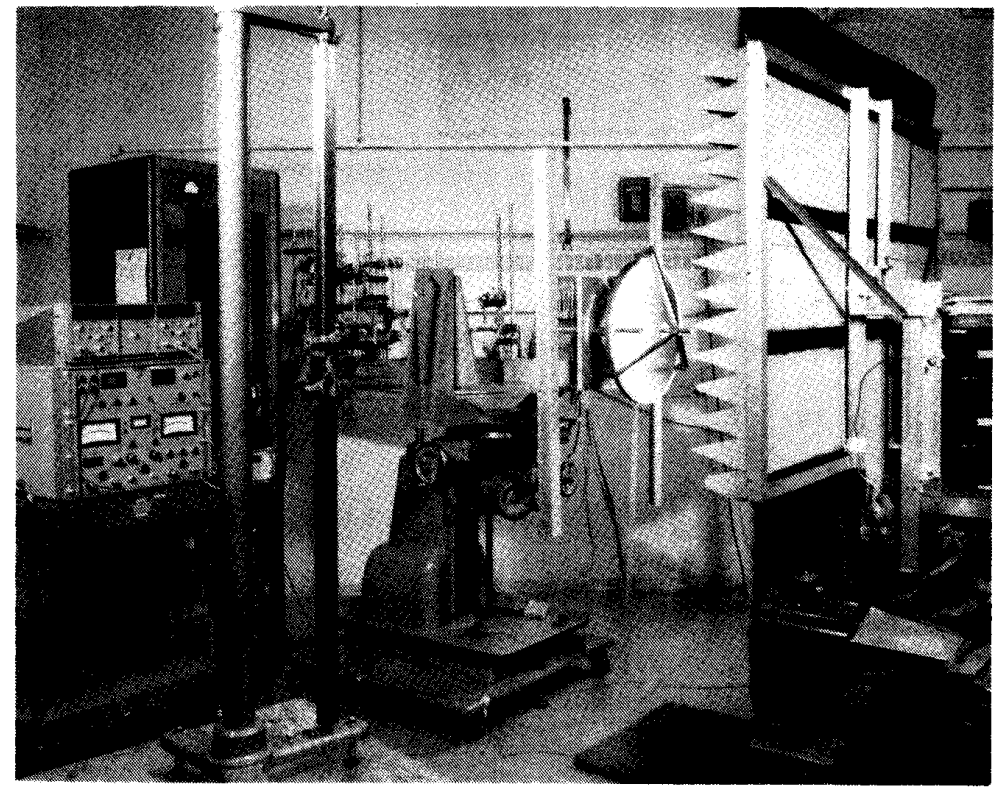

Fig. 2. Measurement system used to perform first planar near-field measurements at NBS.

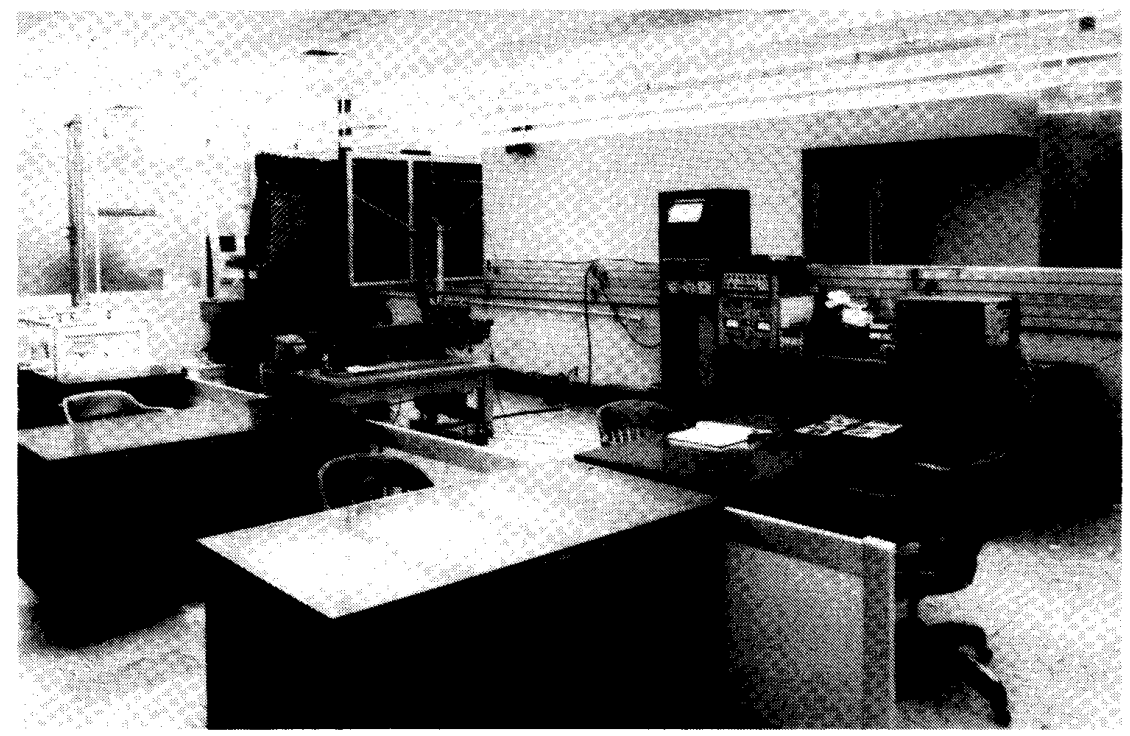

Fig. 3. Array antenna under test in former office area. Note small amount of absorber used.

data at approximately half-wavelength spacings would produce accurate results. Measurements on the millimeter-wave antennas demonstrated this fact. During this period, the fast Fourier transform (FFT) replaced less efficient algorithms.

This small measurement system was also used for the first near-field measurements on a phased array antenna. In 1972 work was begun in cooperation with the Air Force Avionics Laboratory (AFAL) at Wright Patterson Air Force Base, OH, to develop planar near-field measurement techniques for airborne phased arrays. Initially, a fixed-beam array was measured, and one result demonstrated the feasibility of detecting and locating individual faulty elements. Operating phased arrays were difficult to obtain for measurement programs, and when one did become available for a short period in June 1973, it could not be shipped to Boulder, CO The tests were therefore carried out at a laboratory in California, and the NBS measurement system was assembled at that site. As shown in Fig. 3, the antenna measurements were performed in a former office area and received considerable attention from passersby. This project confirmed that this type of measurement system required much less space than other approaches. The data from these measurements [12] were used to extend previous results related to data-point spacing and scan area to phased arrays, and to develop tests to evaluate errors due to multiple reflections.

Further work with phased arrays was carried out in 


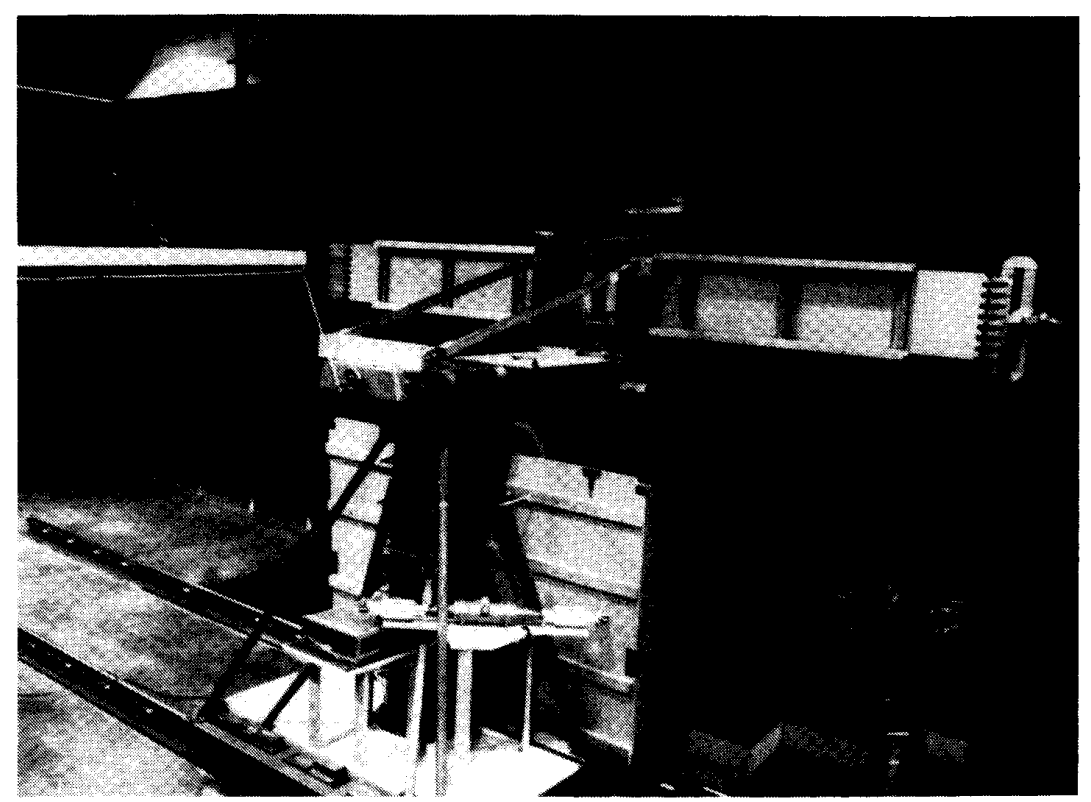

Fig. 4. Ultra-low sidelobe array under test at NBS showing extension of scan area with rails parallel to $x-y$ scanner.

cooperation with the U.S. Navy to support the Aegis radar system. NBS was asked to determine the feasibility of measuring the antenna used in this system using near-field techniques. Accuracy of results was a primary concern, so a detailed error analysis was undertaken to determine the effects of measurement errors on far-field results. Analytical expressions that predicted upper bound errors were derived [13] and were also used to specify the requirements of the measurement system. The positional accuracy of the mechanical scanner and linearity of the receiver were two of the parameters studied. The near-field technique was adopted and is still being used for alignment and testing of the Aegis phased array antennas. The error analysis begun with this study has continued in almost every measurement program at NBS. Further tests and approaches to obtain reliable estimates of uncertainty in the results of planar near-field measurements have been developed. The results of these studies are reported elsewhere in this issue [14].

In 1974, work was completed on a larger planar scanner that would be used on numerous research projects and measurement programs. The mechanical scanner had a measurement area of $4 \mathrm{~m}$ by $4 \mathrm{~m}$, and maximum position error of $0.1 \mathrm{~mm}$. It has served as the design model for a number of other similar scanners, and experience from its use has been applied in most of the current operating near-field systems.

In 1976, research to apply near-field techniques to communication satellite antennas was begun. An engineering model of a commercial satellite antenna was brought to NBS and many tests were performed. Of particular interest was the measurement of shaped beam patterns and the use of dual mode circularly polarized probes to obtain accurate cross polarized patterns. In the years that followed, techniques to measure the combined performance of the antenna along with the transmitter and receiver were developed. Effective isotropic radiated power and saturating flux density can now be obtained from near-field measurements; these techniques are being applied in the design and testing of communication satellite antennas.

In 1985 the measurement facility was modified to accommodate larger antennas. Fig. 4 shows the present configuration with a large slotted waveguide array mounted on the movable antenna mount. The mount moves on a set of rails that are aligned parallel to the scan plane. The test antenna is located in three positions along the rails, and at each position the scanner measures over a $4 \mathrm{~m}$ by $4 \mathrm{~m}$ area. The three segments of data are combined in the computer to achieve a scan area up to $11 \mathrm{~m}$ long and $4 \mathrm{~m}$ high. Measurements on the antenna shown in Fig. 4 demonstrated that the near-field technique could be used to measure very low sidelobes at levels -50 to $-60 \mathrm{~dB}$ below the beam peak.

One of the most rewarding aspects of the near-field work has been the extensive interaction it has produced with other organizations and scientists. These interactions have led to broad application as well as increased development of the near-field measurement technique. In the early days of the work the technique met with considerable skepticism. Many antenna engineers were accustomed to seeing the antenna patterns directly from far-field measurements and were skeptical of the near-field approach, which involved extensive theory and computer processing of large amounts of data. Their feelings were summed up by a comment from one engineer who said, "Near-field measurement won't work; I know it won't work because I tried it and it didn't work." NBS had a major job, then, not only of developing the technique scientifically, but also of educating the antenna community to the value and validity of the technique and of transferring the technology where it could be profitably applied. During the spring of 1964 the PNFS theory was the subject of a joint NBS-University of Colorado graduate course, "Topics in 
Antenna Theory,', taught by Kerns. This course was repeated several times from 1964 to 1973 , each time being more complete as later developments were included. The theory was also included as a topic in a 1966 NBS Precision Measurements Seminar [15]. Short courses devoted entirely to nearfield measurement techniques were given at NBS in 1975, 1977, and 1985, and many seminars and short courses have been given at organizations involved in antenna measurements.

\section{Nonplanar Near-Field Scanning at NBS}

While NBS was a pioneer institution in planar scanning, the NBS contributions in the area of nonplanar scanning resulted primarily from improvements on techniques which were actually developed at other institutions. We discuss the work which was performed in the two practical nonplanar geometries; spherical and cylindrical.

\section{A. Spherical Scanning}

The problem of probe corrected near-field scanning on a spherical surface was first solved completely in 1970 by Jensen in his doctoral dissertation at the Technical University of Denmark (TUD) [16]. His solution was rigorous mathematically but required the inversion of large matrices. This resulted in very inefficient data processing since the time for this technique is proportional to $a_{\lambda}^{6}$ where $a_{\lambda}$ is the radius of the antenna under test in units of the operating wavelength. Even on today's largest computers, the task is so formidable as to require weeks of computation time for antennas of practical size.

Wacker of NBS developed a technique employing orthogonalities; it drastically reduced the computation to a time proportional to $a_{\lambda}^{3}[17]$. In addition, Jensen's theory required not two scans as in the planar and cylindrical case but many scans, each with a different orientation of the probe depending on its complexity. It was realized that if a probe could be built with a high degree of symmetry the many measurements required could be reduced to two. Such probes would have an azimuthal index ${ }^{1} \mu=0$ or $\mu= \pm 1$. If $\mu=0$, the probe measures primarily the radial component of either the electric or magnetic field; if $\mu= \pm 1$, the probe measures a transverse component. Since radial fields decay as $1 / r^{2}$ rather that $1 / r$, this left the $\mu= \pm 1$ probes as the logical candidate. Such probes can be built if they have circularly cylindrical symmetry and are excited with the $\mathrm{TE}_{11}$ mode, the dominant mode in a cylindrical waveguide. Thus the two fundamental contributions which made spherical scanning practical were the new analysis which used orthogonalities and a special probe to solve the probe orientation problem.

The first actual computer program to perform probecorrected spherical analysis was written by Lewis in 1975. In addition to implementing the theory, it was necessary to modify the code for the FFT to transform data arrays that could not all reside in memory at once and, in addition, were not limited to arrays which were an integral power of two in size. Modern virtual memory computers allow this type of

\footnotetext{
The azimuthal index is the index of a Fourier expansion of the probe response which describes the dependence of the response of the probe on rotation about its axis.
}

computation to be done without special user developed software.

The spherical scanning work at NBS was supported initially by AFAL during the years 1973-1977. The first experimental work at NBS consisted of comparisons between patterns computed from planar and spherical near-field scans for a 1700-element constrained lens array supplied by AFAL. Comparisons between far-field patterns computed from planar near-field data and spherical near-field data were made with good agreement.

This work was discussed by Wacker in the Near-Field Measurement Short Course taught at NBS in the summer of 1975. Two of the attendees, Larsen (then a graduate student at TUD) and Hess subsequently made significant contributions to spherical scanning at their own institutions [18], [19].

Larsen obtained the software and with subsequent improvements and modifications employed it in the successful TUD spherical near-field measurement program. Hess also obtained the software and modified it for use on a minicomputer. This software is now used on a commercial spherical near-field measurement system.

Following the conclusion of the work sponsored by AFAL, NBS did not actively engage in research relating to spherical scanning until work relating to simplifications envisioned by Yaghjian and supported by the Office of Naval Research was begun in 1983. The principal investigator for this work was Wittmann. He and Yaghjian, who had since moved to Rome Air Development Center (RADC), Hanscom AFB, have collaborated over the past several years on various aspects of the problem. The probe correction was reformulated so that the probe correction now appears in a similar fashion to that of the planar and cylindrical cases as a modification of the modal coefficients which are calculated for the antenna as though no probe correction were employed [20]. The same program can thus be used for the analysis of data with and without probe correction. A further improvement was also made by performing an integration by parts on the orthogonality relationship which determines the modal coefficients resulting in a savings of about a factor of two [21], [22]. This also permits the same algorithm to be employed to perform the orthogonality integral for both polarizations as well as for the scalar (acoustic) case. Since 1985 the work has been supported by RADC.

Currently, NBS is adding a new spherical coordinate pattern range to its facility to permit both probe pattern measurements and spherical near-field measurements to be performed.

\section{B. Cylindrical Scanning}

The first paper on cylindrical probe-corrected near-field scanning was written by Brown and Jull as part of Jull's dissertation [23], which solved the problem in two dimensions, that is, with no $z$ dependence. While this seminal paper was the first to solve the probe-correction problem in any geometry, practical problems require the use of a threedimensional theory. The first three-dimensional solution to probe-corrected cylindrical near-field scanning was presented by Leach and Paris [24]. Because of the availability of a scanning facility and the relative simplicity of the theory (as compared to spherical), we decided to pursue a modest effort in cylindrical scanning at NBS. Yaghjian reformulated the 
problem using the source scattering matrix approach [25], which allowed the probe-correction coefficients to be calculated separately, as is also the case for planar and spherical. He wrote a program which allowed the calculation of the farfield pattern from data acquired on a cylinder for $z$-polarized fields. While somewhat restrictive in its use, it allowed a number of interesting results to be obtained. Though not a probe-corrected program, it was possible to model the probe correction for $\cos ^{n}$ probe patterns, and this allowed study of some of the effects of probe correction in the nonplanar cases where intuition was not developed as it was for planar scanning.

In addition, Yaghjian's Technical Note [25] contained a new simplified technique for calculating the translated probe coefficients and showed a one-to-one correspondence between a given coefficient and the value of the probe's far-field pattern in a specific direction, much as is the case for planar scanning. The only restriction for this asymptotic form to be valid is that the center of the scan cylinder must lie in the far field of the probe, not a restrictive assumption for almost all practical scanning situations. The complete three-dimensional probe-corrected theory was incorporated in a program written in 1977 by Stubenrauch, who built on Yaghjian's original program.

Experimental comparisons at NBS were first made using the constrained lens array also used for the spherical work with good results. Comparisons have also been made between patterns calculated using planar and cylindrical near-field scanning for a 1.2-m diameter reflector antenna operating at 4 $\mathrm{GHz}$. Cross-polarized patterns also showed agreement with planar results down to the $-40-\mathrm{dB}$ level.

An interesting application of cylindrical scanning was the hybrid scanning technique. In this application, the main beam of a directive antenna is oriented along the $z$ axis of the scan cylinder. Data which permit calculation of the sidelobe region of the antenna as far as $150^{\circ}$ off the main beam direction are acquired. The pattern in the forward direction is computed from planar scanning or cylindrical scanning with the main beam normal to the scan axis (the usual configuration) [26]. Results in the overlapping region of the two scans show good agreement.

\section{ACKNOWLEDGMENT}

NBS has enjoyed a position of leadership in the development of near-field antenna measurement techniques. This has been in part due to a fortituitous set of circumstances but also to the efforts of many of its staff not mentioned in the preceding sections. We wish also to recognize the contributions of D. R. Belsher, R. R. Bowman, J. V. Cateora, M. H. Francis, J. W. Greene, W. E. Jessen, S. B. Kilgore, D. P. Kremer, M. Lojko, A. G. Repjar, and D. T. Tamura.

\section{REFERENCES}

[1] R. C. Baird, "A microwave measurement of the speed of light using a Fresnel-zone Michelson interferometer," Ph.D. dissertation, Univ. Colorado, Boulder, 1965.

[2] _ , "RF measurements of the speed of light," Proc. IEEE, vol. 55 pp. 1032-1039, June 1967

[3] D. M. Kerns and E. S. Dayhoff, "Theory of diffraction in microwave interferometry," J. Res. Nat. Bur. Stand, vol. 64B, pp. 1-13, Jan.Mar. 1960.

[4] D. M. Kerns, "Analytical techniques for the correction of near-field antenna measurements made with an arbitrary but known antenna," presented at the URSI-IRE Meeting, Washington, DC, Apr. 29-May 2, 1963.

[5] - " "Correction of near-field antenna measurements made with an arbitrary but known measuring antenna," Electron. Lett., vol. 6, pp. 346-347, May 28, 1970.

[6] - "New method of gain measurement using two identical antennas," Electron. Lett., vol. 6, pp. 348-349, May 28, 1970.

[7] R. C. Baird, A. C. Newell, P. F. Wacker, and D. M. Kerns, "Recent experimental results in near-field antenna measurements," Electron. Lett., vol. 6, pp. 349-351, May 28, 1970.

[8] P. F. Wacker, "Theory and numerical results for accurate extrapolation of near-zone antenna and scattering measurements," NBS Report (unpublished), Mar. 1972.

[9] A. C. Newell and D. M. Kerns, "Determination of both polarization and power gain of antennas by a generalized 3-antenna measurement method," Electron. Lett., vol. 7, pp. 68-70, Feb. 11, 1971.

[10] A. C. Newell, R. C. Baird, and P. F. Wacker, "Accurate measurement of antenna gain and polarization at reduced distances by an extrapolation technique," IEEE Trans. Antennas Propagat., vol. AP-21, pp. 418-431, July 1973.

[11] D. M. Kerns, Plane-Wave Scattering-Matrix Theory of AntennaAntenna Interactions. Nat. Bureau Standards, Monograph 162, June 1981.

[12] A. C. Newell and M. L. Crawford, "Planar near-field measurements on high performance array antennas," NBSIR 74-380, July 1974.

[13] A. D. Yaghjian, "Upper-bound errors in far-field antenna parameters determined from planar near-field measurements," NBS Technical Note 667, Oct. 1975.

[14] A. C. Newell, "Error analysis techniques for planar near-field measurements," IEEE Trans. Antennas Propagat., pp. 754-768, this issue.

[15] D. M. Kerns, "Antenna measurements with arbitrary antennas at arbitrary distances," contribution in High-Frequency and Microwave Field-Strength Seminar, NBS Report (unpublished), 1966.

[16] F. Jensen, "Electromagnetic near-field far-field correlations," Ph.D. dissertation, Tech. Univ. Denmark, Lyngby, July 1970.

[17] P. F. Wacker, "Non-planar near-field measurements: Spherical scanning," NBSIR 75-809, June 1975.

[18] F. H. Larsen, "Probe-corrected spherical near-field antenna measurements," Ph.D. dissertation, Tech. Univ. Denmark, Lyngby, Dec. 1980.

[19] D. W. Hess and J. J. Tavormina, "Verification testing of a spherical near-field algorithm and comparison to compact range measurements," in Int. IEEE/AP-S Symp. Digest, Los Angeles, CA, June 1981.

[20] A. D. Yaghjian and R. C. Wittmann, "The receiving antenna as a linear differential operator: Application to spherical near-field scanning," IEEE Trans. Antennas Propagat., vol. AP-33, pp. 11751185, Nov. 1985

[21] R. C. Wittmann, "Probe correction in spherical scanning, viewed as an ideal probe measuring an effective field," in Dig. Int. Symp. Antennas Propagat. Boston, MA, June 1984, pp. 674-677.

[22] R. L. Lewis and R. C. Wittman, "Improved spherical and hemispherical scanning algorithms," IEEE Trans. Antennas Propagat., vol. AP-35, pp. 1381-1388, Dec. 1987.

[23] J. Brown and E. V. Jull, "The prediction of aerial radiation patterns from near-field measurements," Proc. Inst. Elec. Eng., vol. 108B. pp. 635-644, Nov. 1961.

[24] W. M. Leach, Jr., and D. T. Paris, "Probe-compensated near-field measurements on a cylinder," IEEE Trans. Antennas Propagat. vol. AP-21, pp. 435-445, July 1973.

[25] A. D. Yaghjian, "Near-field antenna measurements on a cylindrical surface: A source scattering-matrix formulation," NBS Tech. Note 696, Sept. 1977

[26] C. F. Stubenrauch and A. C. Newell, "Some recent near-field antenna measurements at NBS," Microwave J., vol. 23, pp. 37-42, Nov, 1980 .

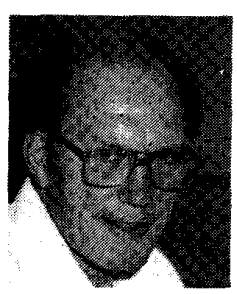

Ramon C. Baird (SM ${ }^{6}$ 6) was born in Ogden, UT, on May 25, 1929. He received the B.S. and M.S. degrees in physics from Brigham Young University, Provo, UT, in 1955 and 1957 , respectively, and the Ph.D. degree in physics from the University of Colorado, Boulder, in 1965.

He joined the National Bureau of Standards' Boulder Laboratory in 1957 and immediately began work on precision millimeter-wave measurements. He was responsible for the development of two refractometers for determining the refractive indi- 
ces of atmospheric gases to accuracies of a few parts in $10^{\circ}$ and, concurrently, the development of a millimeter-wave analogue of the Michelson Interferometer for precision measurements of the speed of light. In 1965 he became Chief of the Antenna Systems Metrology Group of NBS. This Group pioneered the development of the theory and measurement techniques for determining the characteristics of antennas by planar near-field scanning and is currently involved in extending the theory and applications of near-field scanning. Other work of this Group includes the development and maintenance of the primary thermal noise standards at NBS and the development of precision methods for measuring the $\mathrm{G} / \mathrm{T}$ of Earth Terminals. In July 1985, he was appointed Deputy Chief of the NBS' Electromagnetic Fields Division, and he has served as Chief of this Division since April 1987. The division conducts basic and applied research on the interaction of electromagnetic radiation with devices and materials, both in transmission lines and for radiated fields, with emphasis on coherent radiation and applies the results of these investigations for the improvement of measurement science needed to develop, transfer and exploit technology for public benefit. The work exmphasizes the development and evaluation of measurement methods and standards needed to characterize and optimize the mechanisms and output of electromagnetic sources, receivers, passive and active devices, and components. Further, these measurement methods and standards are adapted to establish techniques for measuring crucial properties of matter and electromagnetic fields, and also performance parameters of devices, components, and systems.

Dr. Baird is a member of Phi Kappa Phi and URSI, and a Past Chairman of U.S. URSI Commission A.

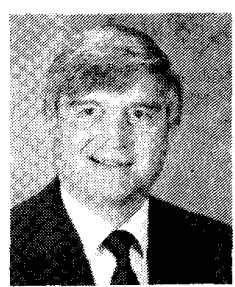

Allen C. Newell (M'73-M'81-SM'83) received the B.S. degree in physics from Brigham Young University, Provo, UT, in 1960, and has done graduate work in electrical engineering at the University of Colorado. Boulder.

He joined the NBS Boulder Laboratories in 1960 and performed research in high-frequency voltage and power measurements. His major scientific interest and experience have been in antenna nearfield theory and measurements and millimeter-wave techniques. Since 1962 he has been involved in a variety of microwave and millimeter-wave measurement problems. He codeveloped a millimeter-wave refractometer accurate to within a few parts in
$10^{8}$, and received a special award for his contributions to a millimeter-wave velocity of light experiment. Beginning in 1965, he co-developed the NBS planar near-field measurement technique for determining antenna characteristics from measurements made close to the antenna. He also co-developed the generalized threc-antenna extrapolation method for performing high-accuracy gain and polarization measurements of microwave antennas. He has published several reports and papers in the above areas and has taught seminars and short courses on antenna measurements at NBS, Georgia Institute of Technology, California State Northridge, and The Technical University of Denmark. He is an internationally recognized expert in the area of near-field antenna measurements. Presently, he serves as a Group Leader for microwave and millimeter-wave antenna measurements in the Antenna Metrology Section of the NBS Electromagnetics Division.

Mr. Newell has served for a number of years on the IEEE Antenna Standards Committee.

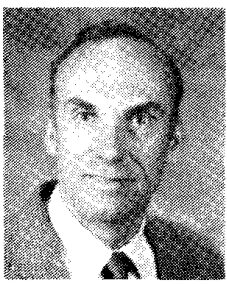

Carl F. Stubenrauch (S`61-S'69-M'71-SM'83) received the B.S. and M.S. degrees from the University of Illinois, Urbana, in 1962 and 1964 and the $\mathrm{Ph} . \mathrm{D}$. from the University of Michigan. Ann Arbor, in 1972, all in electrical engineering

He has served as Instructor in Electrical Engineering at Michigan Technological University, Houghton. MI, and as Assistant Professor of Phys. ics and Mathematics at Concordia Lutheran Junior College, Ann Arbor, MI. He is an Electronics Engineer in the Electromagnetic Fields Division of the National Bureau of Standards. Boulder, CO, where for the past 15 years he has worked in the area of near-field antenna measurements. During this time he has done research on cylindrical and spherical near-field scanning, the extrapolation technique for precision gain measurements. and the calculation of mutual coupling between cosited antennas.

Dr. Stubenrauch is a Registered Professional Engineer. He is a member of URSI Commission A, the Optical Society of America, Sigma Xi. Tau Beta Pi, and Eta Kappa Nu and recently completed a term as an Associate Editor of Radio Science. 